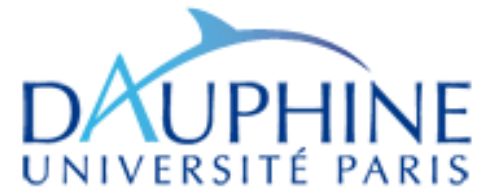

\section{$\mathrm{D}$}

I $\underset{A}{\text { L }}$

UMR 225 IRD - Paris-Dauphine

Institut de recherche pour le développement

DOCUMENT DE TRAVAIL

DT/2014-03

\title{
Le marché du travail en Haïti après le séisme : quelle place pour les jeunes?
}

François ROUBAUD

Constance TORELLI

Claire ZANUSO 


\title{
LE MARCHE DU TRAVAIL EN HAÏTI APRES LE SEISME : QUELLE PLACE POUR LES JEUNES ?
}

\author{
François Roubaud \\ IRD, UMR DIAL, 75010 Paris \\ PSL, Université Paris-Dauphine, LEDa, 75016 Paris, \\ roubaud@dial.prd.fr \\ Constance Torelli \\ INSEE, UMR DIAL \\ constance.torelli@insee.fr \\ Claire Zanuso \\ PSL, Université Paris-Dauphine, LEDa, 75016 Paris, \\ UMR DIAL, 75010 Paris \\ zanuso@dial.prd.fr
}

Document de travail UMR DIAL

Janvier 2014

\section{Résumé}

En janvier 2010, Haïti était touché par le plus terrible tremblement de terre de son histoire tourmentée, suivi d'une mobilisation internationale sans précédent. Depuis lors, une succession de catastrophes naturelles se sont abattues sur le pays (inondations, épidémies, etc.). A l'heure de la commémoration du 4ème anniversaire du séisme, nous nous interrogerons sur la place et le rôle joués par les jeunes dans ce contexte d'exception. L'accent porte sur l'insertion de ces derniers sur le marché du travail, étant entendu que l'emploi est de loin la principale source de revenu des populations, permettant en particulier la survie des plus pauvres. La description fouillée de leur situation sera guidée par un fil rouge : les jeunes ont-ils été épargnés, relativement à leurs aînés, que ce soit grâce aux solidarités familiales ou via l'aide internationale, ou au contraire, ont-ils été sacrifiés en tant que "maillon faible" générationnel. Pour étayer nos analyses, nous mobiliserons la première enquête socio-économique post-séisme à couverture nationale, réalisée fin 2012 par l'Institut Haïtien de Statistique avec l'appui scientifique des auteurs, et dont les résultats sont encore inédits. Pour étudier la dynamique, ces derniers seront confrontés à ceux issus d'une enquête similaire conduite en 2007 par les mêmes partenaires. Nos résultats montrent que les jeunes, et ceux d'origine modeste au premier chef, ont été les principales victimes de l'ajustement à la baisse provoqué par le séisme. La dégradation brutale du sort qui leur est fait est non seulement inique mais elle comprend le développement d'Haïti à moyen terme.

Mots Clés : $\quad$ Marché du travail, Catastrophes naturelles, Jeunes, Haïti, Inégalités, origine sociale.

\begin{abstract}
In January 2010, Haiti has been hit by the worst earthquake in its dramatic history. It was followed by an unprecedented international mobilization. Since then, a succession of natural disasters has struck the country (floods, epidemics, etc.). At the commemoration of the fourth anniversary of the seism, we examine the place and the role played by the Youth in this context of exception. This paper focuses on the insertion of the Youth on the labour market. Labour markets are the transmission belt between the macroeconomic dynamics and household living conditions, as the job earnings constitute the main households resources in developing countries, especially the poor. The thorough analysis of their situation is guided by a key thread: have they been spared, compared to their elders, whether through family solidarity or through international assistance; or conversely, have the Youth been sacrificed as the generational "weak link". Our analysis is based on the first socioeconomic survey post-earthquake. Conducted at the national level the last quarter of 2012 by the National Statistics Office of Haiti with the scientific support of the authors, the results are available for the first time. To study the dynamics, they are compared with those from a comparable survey conducted in 2007 by the same partners. Our results show that young people, and those from disadvantaged social origin in the first place, were the main victims of the downward adjustment caused by the earthquake. The sharp deterioration of their labour market conditions is not only iniquitous but it also compromises the development of Haiti in the medium term.
\end{abstract}

Key words: Labour market, natural disasters, Youth, Haiti, inequality, social origin.

JEL Code:. J13, J21, D1, I20, Q54 


\section{Introduction}

En janvier 2010, Haïti était touché par le plus terrible tremblement de terre de son histoire tourmentée, suivi d'une mobilisation internationale sans précédent. Depuis lors, une succession de catastrophes naturelles se sont abattues sur le pays (inondations, épidémies, etc.). A l'heure de la commémoration du 4ème anniversaire du séisme de 2010, nous nous interrogeons sur la place et le rôle joués par les jeunes dans ce contexte d'exception. L'accent porte sur l'insertion de ces derniers sur le marché du travail, étant entendu que l'emploi est de loin la principale source de revenu des populations, permettant en particulier la survie des plus pauvres. La description fouillée de leur situation est guidée par un fil rouge : les jeunes ont-ils été épargnés, relativement à leurs aînés, que ce soit grâce aux solidarités familiales ou via l'aide internationale, ou au contraire, ont-ils été sacrifiés en tant que "maillon faible" générationnel. D'un point de vue plus général, on se souvient que les printemps arabes ont été déclenchés par l'immolation de Mohamed Bouazizi, un jeune vendeur ambulant tunisien qui ne supportait plus le harcèlement de la police. Dans le contexte de pauvreté généralisée en Haïti, un accès à l'emploi particulièrement difficile pourrait également entrainer la jeunesse dans la violence et provoquer des troubles sociaux.

Pour étayer nos analyses, nous mobilisons la première enquête socio-économique post-séisme à couverture nationale, réalisée fin 2012 par l'Institut Haïtien de Statistique et d'Informatique avec l'appui scientifique des auteurs, et dont les résultats sont encore inédits.

Pour étudier la dynamique, ces derniers sont confrontés à ceux issus d'une enquête similaire conduite en 2007 par les mêmes partenaires.

Cet article se compose de quatre parties. La première fournit des éléments de cadrage général permettant de mettre notre analyse thématique en contexte, sur les caractéristiques du séisme, les dommages qu'il a occasionnés et les politiques d'urgence et de reconstruction mises en œuvre, sur les différentes veines de littérature portant sur notre objet d'étude, et sur les spécificités de notre approche méthodologique. Dans la deuxième partie nous nous intéressons à l'insertion des jeunes sur le marché du travail et aux conditions d'accès à l'emploi. La troisième partie est consacrée à la qualité des emplois (rémunérations, conditions de travail, niveaux de satisfaction). Enfin, la dernière partie cherche à affiner l'approche purement générationnelle. En distinguant les jeunes suivant leur origine sociale, nous nous efforçons d'apprécier l'impact, potentiellement différencié, du séisme sur leurs trajectoires.

\section{I.- Eléments de cadrage}

\section{Le séisme et ses conséquences directes}

Le séisme qui a frappé Haïti le 12 janvier 2010 figure parmi les désastres naturels les plus meurtriers enregistrés depuis 1990. Les chiffres officiels indiquent qu'il aurait été deux fois plus meurtrier que tous les séismes précédents de même magnitude [Bilham, 2010]. Même si des enquêtes récentes menées par des institutions internationales laissent supposer que le nombre officiel de morts est surévalué, il ne fait aucun doute que les conséquences ont été sévères [Kolbe et al., 2010 ; Schwartz et al., 2011 ; Doocy et al., 2013]. Haïti est non seulement particulièrement vulnérable aux catastrophes mais il est aussi le pays le plus exposé 
de la région. On y dénombre un peu plus de 50 désastres naturels depuis 1900 [Heger et al., 2008]. Le contexte dans lequel survient le séisme est particulièrement défavorable car pendant cette décennie, Haïti a été frappé tous les ans, soit par un ouragan tropical soit par des inondations de grande ampleur. Selon la base de données internationale EM-DAT, ces catastrophes antérieures au séisme ont affecté au total plus d'un million de personnes, pour près de 7000 morts [OFDA/CRED, 2011]. D'autres pays ont subi l'impact de séismes similaires ou plus forts (cf. le cas récent du Chili), mais les conséquences ont été bien plus dramatiques pour Haïti [Cavallo et Noy, 2009]. C'est aussi le cas des chocs climatiques, comme par exemple l'ouragan Ike (2008) qui a frappé Cuba comme Haïti, avec 7 morts à déplorer pour Cuba contre cent fois plus en Haïti (793).

D’une intensité sismique de 7,3 (échelle de Richter), le séisme a touché de plein fouet l'Aire métropolitaine de Port-au-Prince, centre économique du pays où habitent près d'un haïtien sur cinq, sans pourtant épargner le reste du pays. En plus des pertes de vie humaines, les destructions de bâtiments (environ 105000 logements et infrastructures ont été totalement détruits et plus de 208000 endommagés) ont provoqué le déplacement de millions de personnes à travers le pays et dans des camps de déplacés. Sept mois après la catastrophe, 1555 camps temporaires hébergeaient 1,5 million d'individus. En septembre 2013, soit trois ans et demi après le séisme, 172000 personnes vivaient toujours dans 306 camps selon le dernier recensement de l'OIM [CCCM, 2013] et ceux qui sont partis n'ont pas forcément trouvé une solution durable de logement. Les dommages et pertes ont été estimés par la Banque mondiale à environ 8 milliards de dollars ou 120\% du PIB.

Cette catastrophe d'une rare ampleur a touché un pays déjà fragile, soumis aux aléas climatiques et à une forte instabilité politique. Elle a donné lieu à une intervention immédiate de la communauté internationale à travers l'envoi d'équipes de secours sur place, de promesses d'aides financières et de soutien au processus de reconstruction. Malgré cela et les milliards de dollars engagés, la situation tarde à se normaliser. Le PIB par tête a chuté d'environ $7 \%$ en 2010 et a rebondi de 3\% l'année suivante. Cependant, bien que limité en termes macroéconomiques, le choc s'inscrit dans un contexte de décroissance sur longue période. Le dernier Rapport sur le développement humain (PNUD, 2013) souligne que le revenu national brut par habitant (RNB) est en constante baisse depuis plus de 20 ans, perdant $41 \%$ de sa valeur entre 1980 et 2012. Avec un RNB par habitant estimé à 1070 dollars constants de 2005 en 2012, Haïti reste le pays le plus pauvre du continent américain et l'un des plus déshérités du monde. Haïti est également parmi les plus inégalitaires de la planète.

Au niveau démographique, la population haïtienne est jeune, à l'instar de la plupart des pays en développement. En 2012, plus de la moitié des Haïtiens ont moins de 21 ans, et les jeunes âgés de 10 à 24 ans (sujet de cette étude) représentent 33\% de la population totale et près de la moitié $(44 \%)$ de la population active [Roubaud et al., 2013]. Confronté à cette réalité, le Gouvernement d'Haïti semble vouloir faire des jeunes une priorité de l'agenda politique. Par exemple, depuis 2006, le secrétariat à la Jeunesse et au Sport est devenu un Ministère à part entière. Toutefois, le rapport de l'Enquête Mortalité, Morbidité et Utilisation des Services (EMMUS V) de 2012 [Cayemittes, 2013] souligne que l'instabilité et l'insécurité des dernières années affectent la capacité des jeunes à prendre part activement aux choix les concernant dans la société haïtienne. 


\section{Une brève revue de la littérature}

Les questions que nous abordons dans cet article se situent à la croisée de deux champs de recherche : l'impact des catastrophes naturelles et les études centrées sur la jeunesse. Dans les deux cas, cette littérature ne couvre pas le cas spécifique d'Haïti.

Le premier champ a connu ces dernières années, un important regain d'intérêt avec la problématique du réchauffement climatique : cette tendance est amenée à se développer. En effet, l'incidence des catastrophes naturelles sur le bien-être économique s'est accrue de manière inquiétante au cours de la dernière décennie. Des recherches plus spécifiquement axées sur la résilience des pays expliquent les effets dévastateurs des catastrophes sur la croissance économique des pays en développement par le fait qu'elles empêchent l'accumulation du capital physique et humain, tandis que les canaux expliquant ce phénomène restent opaques [Skoufias, 2003 ; Yamauchi et al., 2008 et 2009]. Dans ces travaux sur l'impact des catastrophes naturelles, Muller et al. [2009a et 2009b] souligne que la résilience du marché du travail est cruciale, notamment pour les pauvres qui comptent uniquement sur cette activité pour diversifier leurs revenus. Les études suggèrent que les secours d'urgence devraient prendre en considération la protection du marché du travail afin de faciliter la recherche d'emploi dans d'autres sites [Muller et Osgood, 2009b]. Or, la mise en place de telles politiques nécessite une évaluation précise de l'impact des catastrophes passées afin de mieux s'adapter et prévenir les potentiels désastres à venir.

La disponibilité de la base de données internationale EM-DAT sur les désastres et leurs dégâts couvrant tous les pays du monde depuis 1900 a suscité la plupart des études existantes sur l'impact économique des désastres naturels. Une attention particulière a été accordée aux coûts humains et économiques. Les conclusions de ces travaux étaient attendues : les pays en développement sont plus vulnérables aux désastres, même s'ils ne sont pas davantage exposés que les pays développés. Les canaux à travers lesquels les désastres provoquent des impacts si différents demeurent la «boîte noire » de ces études, même si certaines mettent en avant des facteurs institutionnels (gouvernance, inégalités, etc.). D'autres recherches sur l'évaluation de l'impact des désastres et leur impact sur les ménages s'appuient sur des études de cas. Cependant, la faible taille des échantillons et leur manque de représentativité limitent la portée de leurs conclusions.

Le second champ de littérature est lui aussi en expansion, principalement $\mathrm{du}$ fait des bouleversements induits par le phénomène de la mondialisation et des crises économiques qui l'émaillent de façon récurrente. Nous n'aborderons ici que les travaux en économie, sachant que cette thématique intéresse aussi d'autres sciences sociales. En fait, deux initiatives internationales montrent l'intérêt croissant pour le travail des jeunes. La première, intitulée Understanding Children's Work (UCW) a été lancée en 2000 par le BIT, l'UNICEF et la Banque mondiale. Coordonnée par l'Université de Rome, elle comprend une importante composante de recherche. Elle a ainsi recensé 2042 références scientifiques traitant de ce sujet. Initialement exclusivement consacrée au travail des enfants, elle s'est progressivement tournée vers les liens qu'il entretient avec l'éducation, les migrations, et pour ce qui nous concerne l'emploi des jeunes. Dans la continuité de ce passage progressif des enfants aux jeunes, la Banque mondiale a mis en place en 2008 avec d'autres partenaires (dont le projet 
UCW) une nouvelle initiative : le Global Partnership for Youth Employment. Dédiée à promouvoir l'emploi des jeunes (essentiellement en Afrique et au Moyen Orient), elle a également une forte composante de recherche, à côté d'activité plus pratiques, comme la définition et la mise en place de projets de promotion de l'emploi des jeunes. A la différence $\mathrm{du}$ projet UCW, elle promeut principalement les travaux d'évaluation d'impact, tout particulièrement ceux issus des méthodologies expérimentales. Dans le vaste domaine de la littérature sur ces thématiques, celle qui porte sur l'Afrique sub-saharienne nous parait la plus pertinente. En effet, bien qu'Haïti soit rattaché à la zone géographique Amérique latine et Caraïbes, son niveau de développement et ses structures socio-économiques ressemblent plus à ceux du continent noir.

DIAL [2007] procède à une revue de littérature extensive tandis que l'OCDE [2013] recense et évalue les politiques d'emplois spécifiquement destinées aux jeunes, en ouvrant de nouvelles pistes.

A notre connaissance, la seule étude scientifique existante d'évaluation de l'impact du séisme en Haïti adopte une approche indirecte et macroéconomique [Cavallo et al., 2010]. Elle cherche avant tout à chiffrer le montant pécuniaire total de l'impact du séisme. Le coût économique est estimé à partir du coût observé dans d'autres pays ayant des caractéristiques semblables et ayant subi un désastre naturel d'ampleur similaire. La validité de ce modèle estimé à partir de la base de données internationale sur les désastres et développé par l'université de Louvain, repose sur des hypothèses assez fortes, car l'impact économique dépend aussi de facteurs institutionnels difficiles à quantifier. Ces estimations permettent d'apprécier si l'effort des donateurs de la communauté internationale est loin ou proche des besoins. En revanche, elles n'apportent aucune piste quant aux raisons pour lesquelles les désastres causent des dégâts d'ampleur variables d'un pays à l'autre, ni pour définir quelles seraient les politiques publiques les plus adaptées. C'est pourquoi nous plaidons en faveur d'approches de type microéconomiques afin de mieux comprendre les mécanismes à l'œuvre.

En ce qui concerne les jeunes haïtiens et le marché du travail, la littérature péche aussi tant sur le fond que sur la forme. D'une part, elle ne traite que de thématiques connexes à la nôtre : santé, violence, éducation, travail des enfants [Lunde, 2010]. D'autre part, elle dépasse rarement le stade de littérature grise ou de rapports publiés par des organisations ou ONG internationales. La seule étude que nous avons trouvée, est antérieure au séisme [Justesen et Verner, 2007]. En se basant sur des données relativement anciennes (enquête sur les Conditions de vie en Haïti de 2001), sa principale conclusion est que les jeunes femmes doivent être ciblées en priorité par les politiques car ce sont elles qui sont les plus touchées par l'abandon scolaire et le chômage. Elle pose d'ailleurs question dans la mesure où nous montrerons que le chômage n'est pas le principal problème des jeunes sur le marché du travail.

Cette brève revue de littérature confirme donc l'intérêt de notre problématique, et l'originalité potentielle des résultats, dans ses trois dimensions (et leur combinaison). Un séisme d'une amplitude exceptionnelle, un marché du travail - principale courroie de transmission entre la dynamique macroéconomique et les conditions de vie -, les jeunes (et les enfants) enfin, représentant plus de la moitié de la population avec leur fragilisation qui gage l'avenir d'Haïti. 


\section{Quelques considérations méthodologiques}

Avant de présenter les résultats de nos analyses, quelques considérations et précautions méthodologiques s'imposent. Nos données proviennent de deux enquêtes nationales indépendantes. Elles fournissent deux photographies successives de la jeunesse haïtienne, mais pas un suivi biographique des individus. En second lieu, nous ne prétendons pas estimer rigoureusement l'impact du séisme. Les données dont nous disposons ne le permettent pas. D'une part, entre nos deux points de comparaisons, 2007 et 2012, de nombreux autres évènements ont eu lieu, notamment une succession d'autres désastres naturels. D'autre part et plus généralement, il faudrait pouvoir répondre à la question "quelle aurait été la situation des jeunes si le séisme n'avait pas eu lieu ?", qualifiée dans la terminologie des évaluations d'impact de contrefactuel. Il est difficile d'imaginer un protocole permettant de le savoir. En effet, pour mesurer rigoureusement l'impact du séisme sur les jeunes, il faudrait pouvoir identifier deux groupes de jeunes parfaitement identiques avant la catastrophe, l'un ayant été touché sans que l'autre ne l'ait été. Or d'un côté, partout où elle a sévi, tous ont été affectés ; de l'autre, le fait d'avoir été victime ou non n'est pas aléatoire. Il est déterminé en fonction de la localisation géographique; cette dernière ayant elle-même une influence propre sur la situation des jeunes, indépendamment du séisme.

Néanmoins, bien qu'imparfaites, nos deux enquêtes nous permettent de formuler un diagnostic riche d'enseignements. Nous nous appuierons sur une triple clef de lecture : d'abord pour chaque année, en comparant les jeunes avec les adultes plus âgés ; ensuite en comparant les mêmes classes d'âge d'une période à l'autre ; enfin en combinant les deux approches, à savoir en contrastant l'évolution de la situation des jeunes avec celle des adultes. Ces trois perspectives correspondent terme à terme à des approches classiques avec-sans, avant-après et différence de différence (plus connu en anglais sous les appellations respectives de withwithout, before-after et diff-in-diff). Pour mener nos analyses, nous définissons la jeunesse (ou les jeunes) en termes de classe d'âge [pour une discussion voir Antoine et al., 2011]. Comme il n'existe aucun consensus sur les bornes à retenir, nous avons retenu l'ensemble des individus âgés de 10 à 24 ans. Au sein de ce groupe nous distinguons trois classes d'âge, tant leur situation vis-à-vis du marché du travail diffère. Les 10-14 ans (que nous qualifierons par convention "d'enfants") qui, selon la législation haïtienne, devraient aller à l'école et ne sont pas censés travailler. Nous appellerons "adolescents" les jeunes de 15 à 19 ans : a priori encore chez leurs parents, un nombre croissant quitte l'école pour connaître (en toute légalité) une première insertion sur le marché du travail. Enfin, les "jeunes adultes" ont entre 20 et 24 ans. Cette nouvelle étape du cycle de vie devrait être marquée par la volonté de s'établir dans la vie. Alors que seule une infime minorité poursuive leurs études, ces jeunes adultes sont en quête d'autonomie (matrimoniale, résidentielle et professionnelle). Une dernière remarque méthodologique : les données utilisées sont d'une exceptionnelle qualité pour le contexte haïtien, laquelle s'explique par des procédures très serrées de contrôle. De plus, en dépit de différences dans le questionnaire employé en 2007 et en 2012, ces dernières sont minimes. Dérivées d'une même matrice (les enquêtes 1-2-3), la grande homogénéité dans la formulation des questions assure un très bon niveau de comparabilité. 


\section{II.- L'insertion sur le marché du travail et l'accès à l'emploi}

Par rapport à 2007, le taux d'activité global a augmenté de 9 points de pourcentage. Une telle variation en à peine 5 ans est exceptionnelle, le taux d'activité évoluant en général à un rythme très lent. Un tel résultat pourrait paraître paradoxal dans la mesure où la situation économique du pays s'est dramatiquement dégradée sur la période, notamment du fait du séisme. L'interprétation la plus plausible de ce phénomène est à mettre au compte de l'appauvrissement de la population. Pour tenter de compenser la dégradation de leurs conditions de vie, les familles se voient contraintes de mettre au travail les membres secondaires des ménages dans l'espoir d'obtenir des compléments de revenu. Les jeunes sont concernés au premier chef. Alors que 18\% des 10-24 ans étaient actifs en 2007, ils sont 33\% en 2012 (Tableau 1). L'explosion des taux d'activité touche tout particulièrement les jeunes ruraux, avec une augmentation de 25 points de pourcentage. Cette évolution est d'autant plus dramatique que ce sont principalement les moins de 20 ans, dont les enfants de 10-14 ans qui font les frais de cette stratégie. On notera avec intérêt que la conjoncture provoque un phénomène connu sous l'appellation de flexion des taux d'activité, mais dans un sens exactement inverse à celui observé dans les pays développés. Alors que dans ces derniers l'amélioration de l'environnement macroéconomique entraine un appel d'air pour un certain nombre d'inactifs voulant profiter de l'embellie, ici c'est la crise qui pousse la population vers l'emploi, surtout les jeunes.

Le second effet de la dégradation brutale de l'environnement économique des ménages est l'effondrement du taux de chômage des jeunes. De 37\% en 2007, il passe à 14\% en 2012 . Dans le même temps, ce dernier reste plus ou moins constant autour de $13 \%$ pour les aînés. Il convient cependant de se garder d'interpréter cette baisse du chômage des jeunes comme un indice de l'amélioration de leur situation. En effet, et à l'instar de la montée des taux d'activité, elle traduit le fait que les Haïtiens peuvent de moins en moins se permettre d'attendre d'obtenir un emploi correspondant à leur niveau d'éducation ou à leurs aspirations. Au contraire, ils sont contraints de se mettre au travail, quelles que soient la qualité des emplois qu'on leur propose (pour les travailleurs dépendants) ou qu'ils se créent (pour les travailleurs indépendants), afin de subvenir aux besoins de leur famille. Les jeunes générations ont dû réviser leurs prétentions à la baisse de façon drastique ou bien renoncer à prolonger leurs études, ce qui pourrait avoir des conséquences économiques et sociales négatives (désincitation à se maintenir dans le système scolaire, insatisfaction généralisée, frustrations, montée de la violence, etc.). D'une façon plus générale, en l'absence d'assurance chômage et de prestations sociales institutionnalisées, le chômage n'est pas un bon indicateur du désajustement entre l'offre et la demande de travail.

\section{Tableau 1}

Taux d'activité, de chômage et d'emploi par tranche d'âge 2007, 2012

\begin{tabular}{|c|c|c|c|c|c|c|}
\hline \multirow[b]{2}{*}{2012} & \multicolumn{6}{|c|}{ Tranches d'âge } \\
\hline & 10-14 ans & 15-19 ans & 20-24 ans & 10-24 ans & 25 ans et + & Ensemble \\
\hline Taux d'activité & 19,1 & 31,8 & 49,5 & 33,0 & 75,5 & 56,9 \\
\hline Taux de chômage & 1,1 & 8,8 & 22,9 & 13,9 & 14,2 & 14,1 \\
\hline Taux d'emploi & 18,9 & 29,0 & 38,1 & 28,4 & 64,8 & 48,9 \\
\hline
\end{tabular}




\begin{tabular}{|l|c|c|c||c|c|c|}
\hline Taux d'activité & 1,7 & 14,6 & 44,0 & 18,4 & 70,6 & 47,7 \\
Taux de chômage & 18,9 & 36,6 & 37,3 & 36,5 & 12,8 & 16,8 \\
Taux d'emploi & 1,4 & 9,3 & 27,6 & 11,7 & 61,6 & 39,6 \\
\hline \multicolumn{1}{|c|}{ Evolution } \\
Taux d'activité & $+17,4$ & $+17,2$ & $+5,5$ & $+14,6$ & $+4,9$ & $+9,2$ \\
Taux de chômage & $-17,8$ & $-27,8$ & $-14,4$ & $-22,6$ & $+1,4$ & $-2,7$ \\
Taux d'emploi & $+17,5$ & $+19,7$ & $+10,5$ & $+16,7$ & $+3,2$ & $+9,3$ \\
\hline
\end{tabular}

Sources : EEEI, 2007, IHSI / DIAL ; ECVMAS, 2012, IHSI / DIAL / Banque mondiale ; calculs des auteurs.

La montée de l'activité et l'effondrement du chômage des jeunes se traduit mécaniquement par un accroissement du taux d'emploi. Alors qu'une proportion négligeable des enfants était employée en 2007, ils sont près de $20 \%$ en 2012. La croissance est du même ordre pour les $15-19$ ans (9\% vs 29\%) et un peu plus faible pour les 20-24 ans (28\% vs 38\%). La croissance globale du taux d'emploi provient exclusivement de la mise au travail des jeunes : +17 points pour eux contre une quasi stabilité pour les 25 ans et plus ( +3 points).

Cependant cette insertion accrue et facilitée sur le marché du travail n'est pas nécessairement une bonne chose, en particulier si elle s'accompagne d'une augmentation du travail des enfants d'une part et d'un désinvestissement scolaire de l'autre. Sur le premier point nous avons montré que c'était effectivement le cas. En revanche concernant le second, la figure 1 montre qu'il n'en est rien. Ainsi entre 2007 et 2012, la proportion d'enfants qui n'ont pas été scolarisés est en baisse, tandis que le niveau scolaire atteint est plus élevé, ce quelle que soit la tranche d'âge. Ce résultat, d'autant plus paradoxal qu'il se produit dans un environnement économique dégradé, montre l'attachement indéfectible des familles à l'école. Cet accroissement de la fréquentation scolaire touche aussi bien les garçons que les filles, avec même un léger avantage à ces dernières.

Figure 1 : Niveau d'éducation par tranche d'âge 2007-2012
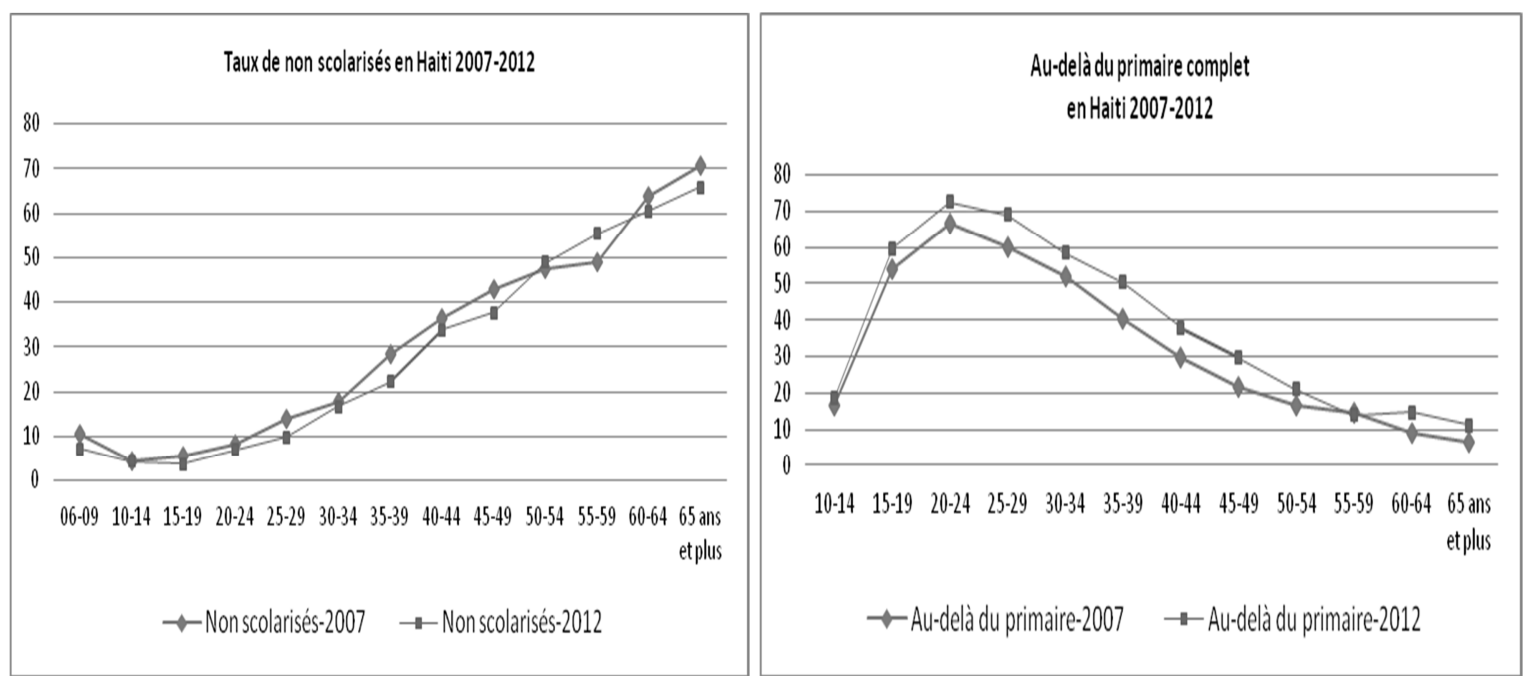

Sources : EEEI, 2007, IHSI / DIAL ; ECVMAS, 2012, IHSI / DIAL / Banque mondiale ; calculs des auteurs.

Evidemment et pour aller plus loin, il faudrait pouvoir mesurer non seulement la quantité mais aussi la qualité de l'éducation. Nous ne disposons malheureusement pas de données directes dans nos enquêtes pour étayer cette question. Mais, il a pu être montré que l'accroissement rapide des taux de scolarisation (de 55\% en 2005 à $77 \%$ en 2012) 
s'accompagne de taux de redoublement, d'entrées tardives et d'abandon scolaire massif [Cayemittes, 2013]. Néanmoins, l'enquête permet d'estimer la proportion de jeunes qui mènent de front étude et emploi et le nombre d'heures qu'ils y consacrent. On peut en effet légitimement supposer que l'exercice d'un emploi nuit à l'attention des jeunes et à la qualité des apprentissages scolaires.

Le tableau 2 montre clairement que la légère augmentation du taux de fréquentation scolaire cache en fait une dégradation des conditions dans lesquelles les jeunes suivent leurs études. D'une part, la proportion de ceux qui étudient tout en travaillant a été multipliée par trois en cinq ans. Cette dégradation est d'autant plus forte qu'on est jeune. Ainsi, alors que $90 \%$ des enfants de 10 à 14 ans se consacraient entièrement à leurs études en 2007, ils ne sont plus que $75 \%$ en 2012. A contrario, la part de ceux qui doivent combiner école et emploi, négligeable en 2007 concerne maintenant $16 \%$ d'entre eux. Le fait que la mise au travail des jeunes se traduise plus par la combinaison école et emploi que par la substitution de l'emploi à l'école est la marque de l'attention que les parents accordent à l'école. La seule bonne nouvelle sur ce front, est que la part des oisifs (ni à l'école, ni en emploi) a un peu diminué.

Plus souvent mobilisés sur plusieurs fronts en même temps, les jeunes consacrent moins de temps à leurs études. La durée de fréquentation scolaire est restée globalement stable pour ceux qui n'exercent pas d'emploi. En revanche, elle a significativement chuté pour les élèves travailleurs. Encore une fois, ce sont les plus jeunes qui sont les principales victimes de cet ajustement à la baisse. Au total, la baisse du nombre d'heures consacrées aux études est la combinaison d'un recul chez ceux qui travaillent en même temps et de la forte poussée des élèves travailleurs, qui étudient structurellement moins que ceux qui se concentrent à plein temps à l'école ; le rapport est en moyenne de un à deux. Ce dernier point confirme l'hypothèse selon laquelle l'exercice d'un emploi est un frein aux apprentissages scolaires.

\section{Tableau 2}

Ecole et emploi des jeunes par tranche d'âge

\begin{tabular}{|c|c|c|c|c|c|c|c|c|}
\hline \multirow[b]{2}{*}{2012} & \multicolumn{4}{|c|}{ Distribution } & \multicolumn{4}{|c|}{ Nombre d'heures consacré aux études } \\
\hline & 10-14 ans & 15-19 ans & 20-24 ans & $10-24$ ans & 10-14 ans & $15-19$ ans & 20-24 ans & 10-24 ans \\
\hline Ecole seule & 74,8 & 59 & 30,1 & 55,4 & 10,5 & 12,0 & 12,2 & 11,3 \\
\hline Ecole et Emploi & 16,2 & 20,4 & 12,1 & 16,4 & 5,4 & 5,8 & 6,6 & 5,9 \\
\hline Emploi seul & 2,6 & 8,2 & 25,8 & 11,8 & - & 0,2 & 0,1 & 0,1 \\
\hline Ni Ecole ni Emploi & 6,4 & 12,4 & 32,0 & 16,4 & - & 0,4 & 0,9 & 0,6 \\
\hline Total & 100 & 100 & 100 & 100 & 8,7 & 8,3 & 4,8 & 7,4 \\
\hline \multicolumn{9}{|l|}{2007} \\
\hline Ecole seule & 90,3 & 73,6 & 37,4 & 68,8 & 11,7 & 11,8 & 11,5 & 11,7 \\
\hline Ecole et Emploi & 1,1 & 4,4 & 9,6 & 4,8 & 10,6 & 7,9 & 6,9 & 7,5 \\
\hline Emploi seul & 0,3 & 5,1 & 18,2 & 7,2 & - & - & - & - \\
\hline Ni Ecole ni Emploi & 8,3 & 16,9 & 34,8 & 19,1 & - & - & - & - \\
\hline Total & 100 & 100 & 100 & 100 & 10,7 & 9,1 & 5,0 & 8,4 \\
\hline \multicolumn{9}{|l|}{ Evolution } \\
\hline Ecole seule & $-15,5$ & $-14,6$ & $-7,3$ & $-13,4$ & $-1,2$ & $+0,2$ & $+0,7$ & $-0,4$ \\
\hline Ecole et Emploi & $+15,1$ & $+16,0$ & $-6,1$ & $+9,2$ & $-5,2$ & $-2,1$ & $-0,3$ & $-1,6$ \\
\hline Emploi seul & $+2,3$ & $+3,1$ & $+7,6$ & $+4,6$ & - & - & - & - \\
\hline
\end{tabular}




\begin{tabular}{|c|c|c|c|c||c|c|c|c|} 
Ni Ecole ni Emploi & $-1,9$ & $-4,5$ & $-2,8$ & $-2,7$ & - & - & - & - \\
\hline Ensemble & - & - & - & - & $-2,0$ & $-0,8$ & $-0,2$ & $-1,0$ \\
\hline
\end{tabular}

Sources : EEEI, 2007, IHSI / DIAL ; ECVMAS, 2012, IHSI / DIAL / Banque mondiale ; calculs des auteurs.

L'exercice d'une activité économique n'est pas le seul à pouvoir constituer un frein à la scolarisation et aux apprentissages. Les tâches domestiques confiées aux jeunes peuvent également peser sur leur disponibilité (en temps et d'esprit). Le diagnostic établi plus haut à propos du temps d'études se voit renforcer lorsque l'on prend en compte l'ensemble des activités réalisées par les jeunes. Les jeunes scolarisés qui ne travaillent pas sont également ceux qui consacrent le moins de temps aux activités domestiques. Ils ne sont néanmoins que partiellement épargnés, puisqu'ils y passent quand même 8 heures par semaine (et 9 heures en 2007), contre 10 heures (et 11 heures en 2007) pour l'ensemble des jeunes (Figure 2).

Figure 2

Emploi du temps des jeunes en 2012 et 2007

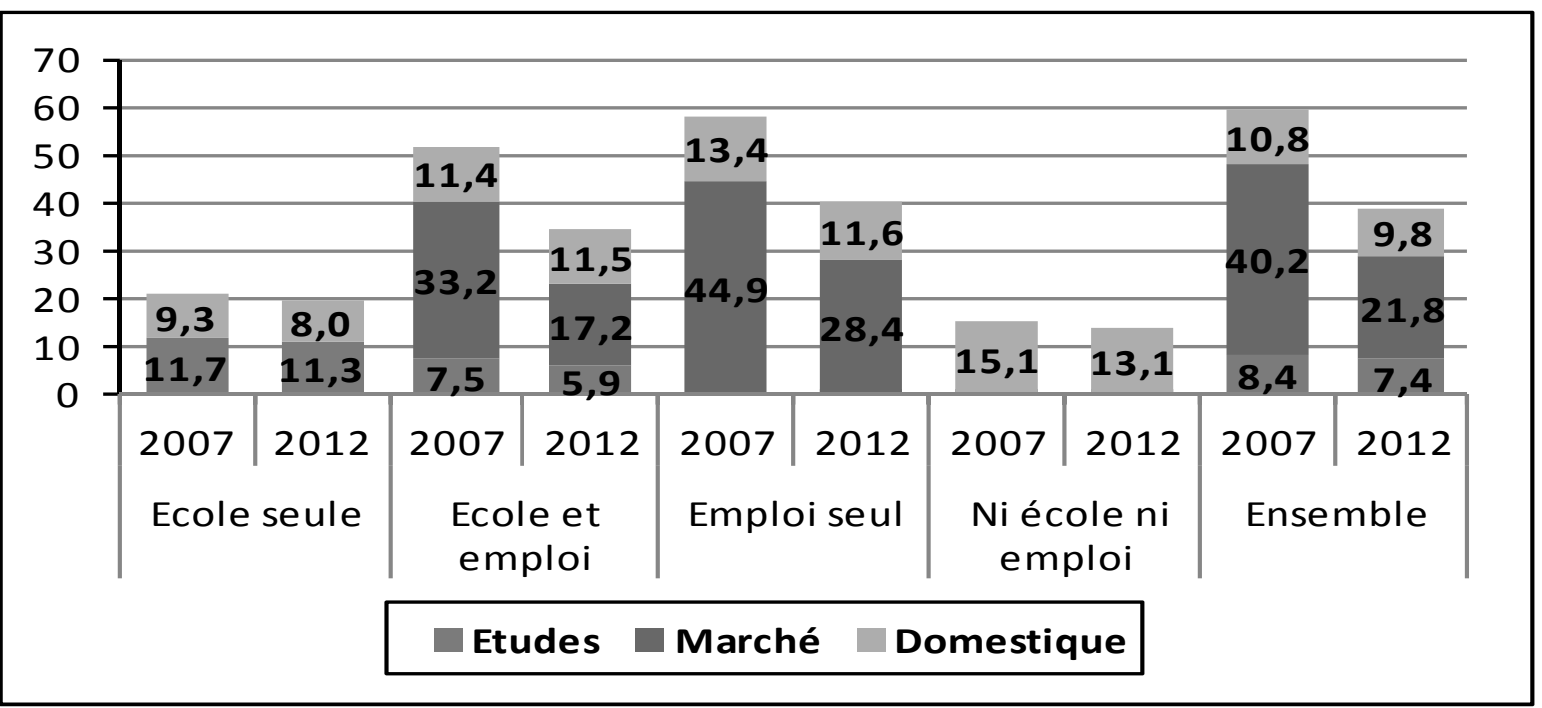

Sources : EEEI, 2007, IHSI / DIAL ; ECVMAS, 2012, IHSI / DIAL / Banque mondiale ; calculs des auteurs.

\section{III.- La qualité des emplois}

Entre 2007 et 2012, l'économie haïtienne a créé des emplois en masse, tandis que le chômage s'effondrait. Les jeunes ont été les principaux "bénéficiaires" de cette dynamique. Néanmoins, nous avons vu qu'il ne s'agissait pas nécessairement d'une amélioration de leur situation, notamment pour les enfants. Une seconde raison potentielle qui pourrait amener à nuancer le caractère positif d'un meilleur accès à l'emploi est qu'il se soit fait au détriment de la qualité des emplois. C'est l'arbitrage classique entre quantité et qualité, appréciée en termes de rémunérations et de conditions de travail, que nous explorons ici. 
Si pour l'ensemble de la population, le taux de salarisation reste constant à $22 \%$, il résulte d'un effet croisé : une chute de 9 points pour les jeunes, compensée par une augmentation du même ordre (+8 points) chez les adultes. Mécaniquement, la proportion d'emplois vulnérables, c'est-à-dire selon le BIT les emplois non salariés, qui était initialement plus faible chez les jeunes (78\% vs $83 \%$ ) est aujourd'hui plus élevée (87\% vs $75 \%$ ). Cette chute de la proportion de salariés chez les jeunes est d'autant plus surprenante que la structure par sousclasses d'âge des statuts dans l'emploi reste constante entre 2007 et 2008. Ce paradoxe apparent s'explique par un effet de structure : afflux massif des plus jeunes qui travaillent quasiment tous comme aides familiaux.

On retrouve des résultats convergents en distinguant les emplois suivant leur secteur institutionnel d'appartenance. Déjà quasiment exclus du secteur formel (privé et public) dès 2007, les jeunes se sont massivement rabattus sur le secteur primaire en 2012, au détriment du secteur informel non agricole. Ce retour à l'agriculture est une régression dans la mesure où il s'agit du secteur le moins productif. La montée de la part de l'emploi agricole dans l'emploi total (+9 points) est surtout dû aux jeunes (+28 points), et seulement marginalement aux adultes (+3 points), ces derniers voyant même leurs emplois se formaliser.

Tableau 3

Statut dans l'emploi par tranche d'âge en 2007 et 2012

\begin{tabular}{|c|c|c|c|c|c|c|}
\hline \multirow[b]{2}{*}{2012} & \multicolumn{6}{|c|}{ Tranches d'âge } \\
\hline & 10-14 ans & 15-19 ans & 20-24 ans & 10-24 ans & 25 ans et + & Ensemble \\
\hline Salariés & 2,8 & 8,0 & 23,3 & 13,2 & 25,0 & 22,0 \\
\hline Indépendants & 4,2 & 14,1 & 25,7 & 16,7 & 60,1 & 49,0 \\
\hline Aide familiaux & 93,0 & 77,9 & 51,0 & 70,1 & 14,9 & 29,0 \\
\hline Ensemble & 100 & 100 & 100 & 100 & 100 & 100 \\
\hline \multicolumn{7}{|l|}{2007} \\
\hline Salariés & 2,3 & 7,1 & 25,9 & 21,9 & 17,3 & 17,9 \\
\hline Indépendants & 4,5 & 14,1 & 25,8 & 57,4 & 75,7 & 73,3 \\
\hline Aide familiaux & 93,2 & 78,8 & 48,3 & 20,8 & 7,0 & 8,8 \\
\hline Ensemble & 100 & 100 & 100 & 100 & 100 & 100 \\
\hline \multicolumn{7}{|l|}{ Evolution } \\
\hline Salariés & $+0,5$ & $+0,9$ & $-2,6$ & $-8,7$ & $+7,7$ & $+4,1$ \\
\hline Indépendants & $-0,3$ & 0 & $-0,1$ & $-40,6$ & $-15,7$ & $-24,3$ \\
\hline Aide familiaux & $-0,2$ & $-0,9$ & $+2,7$ & $+49,3$ & $+8,0$ & $+20,2$ \\
\hline
\end{tabular}

Sources : EEEI, 2007, IHSI / DIAL ; ECVMAS, 2012, IHSI / DIAL / Banque mondiale ; calculs des auteurs.

Les revenus du travail sont à l'évidence un argument déterminant de la qualité des emplois, même s'il n'est pas le seul. Les deux enquêtes permettent de calculer la rémunération mensuelle dans l'emploi principal (y compris primes et avantages divers) aux deux dates. Pour assurer la comparaison en équivalent pouvoir d'achat, les données de 2007 ont été converties aux prix de 2012 en utilisant comme déflateur l'indice des prix à la consommation. Il convient de rappeler que les résultats doivent être interprétés avec précaution, compte tenu de la difficulté intrinsèque à mesurer les revenus dans toutes les enquêtes.

Si l'on prend l'ensemble de la main-d'œuvre y compris les aides familiaux dont la rémunération est nulle, les jeunes gagnent largement moins que les adultes. En 2012, la rémunération mensuelle moyenne est de 1300 gourdes pour les jeunes (Figure 3). Elle 
dépasse 6000 gourdes chez les adultes. Ce résultat peut éventuellement s'expliquer par des différences dans le type d'emplois occupés et les caractéristiques des travailleurs ; seules des équations de gains permettraient de les contrôler. En revanche, les taux de croissance sur 5 ans montrent sans ambigüité que les jeunes ont le plus pâti de la crise. Leur revenu moyen a baissé de $-59 \%$ quand il augmentait de $+23 \%$ chez leurs ainés. L'analyse des rémunérations médianes, moins sensibles aux erreurs de mesure, donne des résultats similaires. Elles sont moins favorables dans l'ensemble, en niveau comme en évolution. Si les adultes ont perdu $29 \%$ de pouvoir d'achat, la chute pour les jeunes est encore plus massive ; plus de la moitié d'entre eux ne bénéficiant d'aucun émolument (en cash ou en nature).

Evidemment, la forte poussée des aides familiaux non rémunérés chez les jeunes pourrait être à l'origine de cette tendance défavorable aux jeunes. Il n'en est rien. Par construction, une fois éliminés les non-rémunérés, le montant des revenus du travail augmentent. Mais la position relative (et absolue d'ailleurs) des jeunes continue à se dégrader fortement (-29\% pour la médiane des 10-24 ans et $-3 \%$ pour la moyenne ; contre respectivement $-20 \%$ et $+18 \%$ pour les plus de 25 ans).

\section{Figure 3}

\section{Rémunération mensuelle réelle par tranche d'âges 2007 et 2012}

y compris aides familiaux non rémunérés

hors aides familiaux non rémunérés
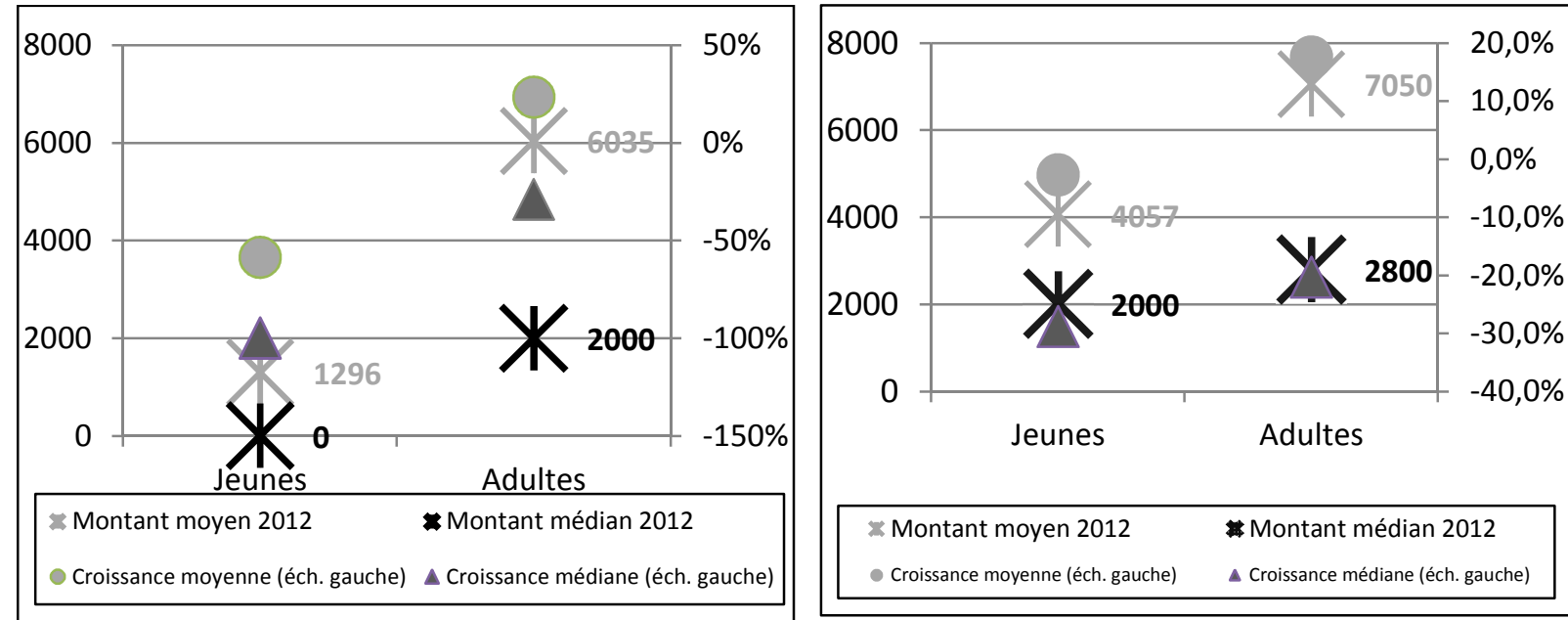

Sources : EEEI, 2007, IHSI / DIAL ; ECVMAS, 2012, IHSI / DIAL / Banque mondiale ; calculs des auteurs.

Au-delà de la rémunération, la qualité des emplois dépend aussi largement des conditions de travail. Le niveau de protection sociale, et partant le taux d'emploi informel (emplois non protégés) est l'indicateur le plus usité dans ce domaine. Cependant, en Haïti, le nombre d'emplois protégés est tellement insignifiant (moins de 5\%), qu'il ne permet pas de hiérarchiser les emplois. D'autres dimensions de la qualité des emplois apparaissent bien plus discriminantes. Sur ce front comme sur celui des revenus, le diagnostic est le même : les jeunes sont à la fois les plus mal lotis et ceux dont la situation a empiré le plus. Par exemple, alors qu'en 2007, 21\% des emplois occupés par les jeunes étaient irréguliers (occasionnels à la tâche, à la journée ou saisonniers), ils étaient $25 \%$ en 2012. Dans le même temps, cette proportion était initialement plus faible (18\%), mais elle a encore reculé (13\% en 2012). Le 
lieu d'exercice de la profession fournit également une mesure indirecte de la pénibilité des emplois et pour les entreprises, de leur productivité potentielle. Nous distinguons trois types d'entreprises par ordre décroissant de précarité : celles qui se développent sans locaux propres et pérennes (in the open sun en anglais), donc soumises aux intempéries telles qu'activités ambulantes ou postes improvisés sur la voie publique ; celles qui sont abritées dans les domiciles, avec ou sans aménagements intérieurs ; enfin celles qui bénéficient d'installations spécifiques et en dur (échoppes, boutiques, ateliers, cabinets, etc.). Dans l'ensemble, la plus grande vulnérabilité règne en maître. Seulement $15 \%$ des jeunes bénéficiaient de locaux professionnels en 2012. Cette faible proportion n'est pas l'apanage des jeunes générations, puisqu'elle est du même ordre chez leurs ainés (17\%). En majorité, les Haïtiens, jeunes comme plus âgés, exercent à l'extérieur (près de 70\%). Cette similitude n'a rien d'étonnant puisque les premiers travaillent dans les mêmes unités que les seconds en temps qu'aides familiaux. Néanmoins, bien que le mouvement soit moins prononcé que pour les revenus, la situation des jeunes se dégrade plus que celle de leurs ainés, avec un recul de 7 points de la part de ceux exerçant des locaux professionnels pour les uns contre 4 points pour les autres.

Nous l'avons vu, si le chômage n'est pas un bon indicateur des tensions sur le marché du travail, ces dernières peuvent être approchées par les différentes formes de sous-emploi, tout particulièrement dans le contexte des pays en développement, où la qualité des emplois importe plus que leur quantité. Reflétant l'aspect multidimensionnel du sous-emploi, trois indicateurs sont couramment utilisés pour appréhender ses différentes composantes.

Le volume horaire de travail permet de quantifier l'importance du sous-emploi lié à la durée du travail (qualifié initialement de sous-emploi "visible", par opposition au sous-emploi "invisible"). Ce concept vise à mesurer l'insuffisance de demande de travail de la part des entreprises. Pour entrer dans cette catégorie, il faut travailler moins que la durée "normale" du travail de manière involontaire. En accord avec la définition internationale établie par le BIT, les actifs occupés doivent donc remplir trois conditions : exercer moins de 35 heures dans leur emploi principal, souhaiter travailler plus et être disponible pour le faire. Cette première forme de sous-emploi des jeunes recule de 16 points contre une quasi-stabilité chez les 25 ans et plus (Tableau 4). Comme pour la baisse du chômage, cette évolution a priori favorable ne l'est pas. Elle signifie simplement que les jeunes ont été obligés de travailler plus longtemps pour subvenir aux besoins de leur famille.

Le sous-emploi lié à la durée du travail ne constitue qu'une modalité à travers laquelle se manifeste le sous-emploi. Ainsi et paradoxalement, l'existence d'horaires de travail excessivement élevés peut, elle aussi, être synonyme de sous-emploi, lorsque cette charge horaire est rendue nécessaire par une productivité du travail anormalement faible : c'est le concept de « sous-emploi invisible ». Nous avons retenu comme indicateur de sous-emploi invisible le pourcentage d'actifs occupés gagnant moins que le salaire minimum, soit 70 gourdes par jour en 2007 et son équivalent en parité de pouvoir d'achat en 2012. Le diagnostic est sans appel, ce qui n'est pas une surprise compte tenu de la dynamique des rémunérations analysée plus haut. Si le sous-emploi invisible des adultes s'accroît sensiblement entre les deux dates, il a presque doublé pour les jeunes (48\% à 83\%), affectant plus des quatre cinquièmes d'entre eux fin 2012. 
Tableau 4

Taux sous-emploi par tranche d'âge 2007, 2012

\begin{tabular}{|c|c|c|c|c|c|c|}
\hline \multirow[b]{2}{*}{2012} & \multicolumn{6}{|c|}{ Tranches d'âge } \\
\hline & 10-14 ans & 15-19 ans & 20-24 ans & 10-24 ans & 25 ans et + & Ensemble \\
\hline Taux sous-emploi visible & 3,7 & 7,8 & 16,0 & 10,2 & 16,4 & 14,8 \\
\hline Taux sous-emploi invisible & 99,9 & 89,2 & 70,5 & 83,8 & 47,2 & 56,5 \\
\hline Taux sous-emploi global & 99,9 & 92,3 & 81,6 & 88,8 & 61,3 & 68,3 \\
\hline \multicolumn{7}{|l|}{2007} \\
\hline Taux sous-emploi visible & 49,8 & 35,1 & 21,3 & 26,4 & 19,6 & 20,5 \\
\hline Taux sous-emploi invisible & 83,6 & 58,7 & 41,8 & 48,4 & 35,2 & 36,9 \\
\hline Taux sous-emploi global & 86,7 & 81,2 & 67,8 & 72,3 & 52,7 & 56,0 \\
\hline \multicolumn{7}{|l|}{ Evolution } \\
\hline Taux sous-emploi visible & $-46,1$ & $-27,3$ & $-5,3$ & $-16,2$ & $-3,2$ & $-5,7$ \\
\hline Taux sous-emploi invisible & $+16,3$ & $+30,5$ & $+28,7$ & $+35,4$ & $+12,0$ & $+19,6$ \\
\hline Taux sous-emploi global & $+13,2$ & $+11,1$ & $+13,8$ & $+16,5$ & $+8,6$ & $+12,3$ \\
\hline
\end{tabular}

Sources : EEEI, 2007, IHSI / DIAL ; ECVMAS, 2012, IHSI / DIAL / Banque mondiale ; calculs des auteurs.

Les différentes composantes du sous-emploi (chômage, sous-emploi lié à la durée du travail et sous-emploi invisible) peuvent être agrégées pour obtenir un indicateur synthétique de sousemploi, appelé sous-emploi global. L'introduction du chômage (qui constitue la forme la plus extrême du sous-emploi, puisque les chômeurs n'ont pas d'emploi et par conséquence ne touchent aucune rémunération) implique que cet indicateur doit être mesuré sur l'ensemble des actifs et non sur les seuls actifs occupés comme pour les deux autres formes de sousemploi. En dépit de la baisse du chômage et du sous-emploi visible, le taux de sous-emploi global des jeunes dérape. La situation est à ce point dramatique que $89 \%$ des jeunes actifs ou trois jeunes sur dix, est d'une façon ou d'une autre en situation de sous-emploi. Ils n'étaient "que" $13 \%$ en 2007.

Pour clore cette partie sur la qualité des emplois, la mobilisation de l'indicateur de satisfaction au travail apparaît particulièrement judicieux. En effet, tous les autres indicateurs sont partiels et ne mesurent qu'une seule dimension (rémunération, pénibilité, vulnérabilité et plus généralement les conditions de travail, protection sociale, etc.). A contrario, la satisfaction dans l'emploi fournit une mesure synthétique de la façon dont les individus eux-mêmes jugent leur emploi, prenant en compte toutes les dimensions pertinentes, qu'elles soient liées au marché du travail ou non. Ainsi par exemple, un emploi bien rémunéré et possédant toutes les caractéristiques d'un "bon emploi", pourra ne procurer qu'une faible satisfaction s'il ne permet pas à une femme (ou plus rarement un homme) de s'occuper de ses enfants, de dégager du temps pour entreprendre des activités domestiques ou de loisirs. De plus, la satisfaction dans l'emploi est un indicateur très facile à appréhender sans erreur de mesure, comme en atteste le très faible taux de non-réponse $(0,3 \%)$. Il va sans dire que ce n'est pas le cas des revenus du travail, qui constituent pourtant l'indicateur le plus utilisé pour apprécier la qualité des emplois. Du côté des points faibles, la satisfaction (comme tous les indicateurs subjectifs) est sensible au phénomène dit "d'attrition des préférences". C'est souvent le cas des plus démunis : leurs normes sont moins exigeantes et leurs aspirations plus modestes. Faute d'avoir été appréhendé en 2007, nous ne pouvons pas apprécier l'évolution, mais seulement la situation en 2012 ; les résultats étant en eux-mêmes riches d'enseignements. 
A un niveau global, deux faits saillants méritent d'être soulignés : la distribution des réponses des jeunes et des adultes est étonnement proche ; le niveau d'insatisfaction est singulièrement élevé. Dans les deux groupes, les deux tiers sont soient "très insatisfaits" ou "plutôt insatisfaits", le solde de satisfaction étant largement négatif (-30 points ; Figure 4). A titre de comparaison, au cœur de la crise financière internationale, le solde était de +31 au Vietnam en 2009.

Lorsqu'on décompose les emplois par secteur institutionnel, quelques différences se font jour. C'est dans le secteur formel que le niveau de satisfaction est le plus élevé, même si le solde est rarement supérieur à zéro (dans le public). A l'autre extrémité de la hiérarchie on trouve le secteur informel et le secteur primaire avec des soldes très dégradés ( -33 et -34 points respectivement). Cette opposition vaut aussi bien pour les jeunes que pour les adultes. Les jeunes qui travaillent dans l'agriculture sont les plus critiques de tous. Leurs homologues adultes sont un peu moins négatifs. On observe une relation inverse dans le secteur informel, les jeunes étant relativement plus heureux au travail. Quant au secteur formel, les écarts sont peu significatifs, vu la faiblesse des effectifs.

In fine, la principale interrogation qui subsiste est pourquoi les jeunes et les adultes affichent un niveau de satisfaction équivalent alors que les premiers occupent les emplois les moins enviables et que leur situation s'est le plus dégradée. Faute d'informations supplémentaires, nous ne pouvons émettre que des hypothèses. La première serait que les jeunes sont moins exigeants que leurs ainés. Une telle interprétation semble peu probable. Les travaux réalisés en Afrique ou au Vietnam montre le contraire [Razafindrakoto et Roubaud, 2013 ; Wachsberger et al., 2012]. La seconde, qui ne reste qu'une conjecture, est que les jeunes trouvent d'autres intérêts à travailler que les caractéristiques de l'emploi lui-même. Dans ce sens, on peut invoquer l'effondrement du taux de chômage des jeunes, qui préfèreraient un emploi de mauvaise qualité à pas d'emploi du tout ; ce qui semble être le cas en général.

Figure 4

Satisfaction dans l'emploi par classe d'âge et origine sociale en 2012 


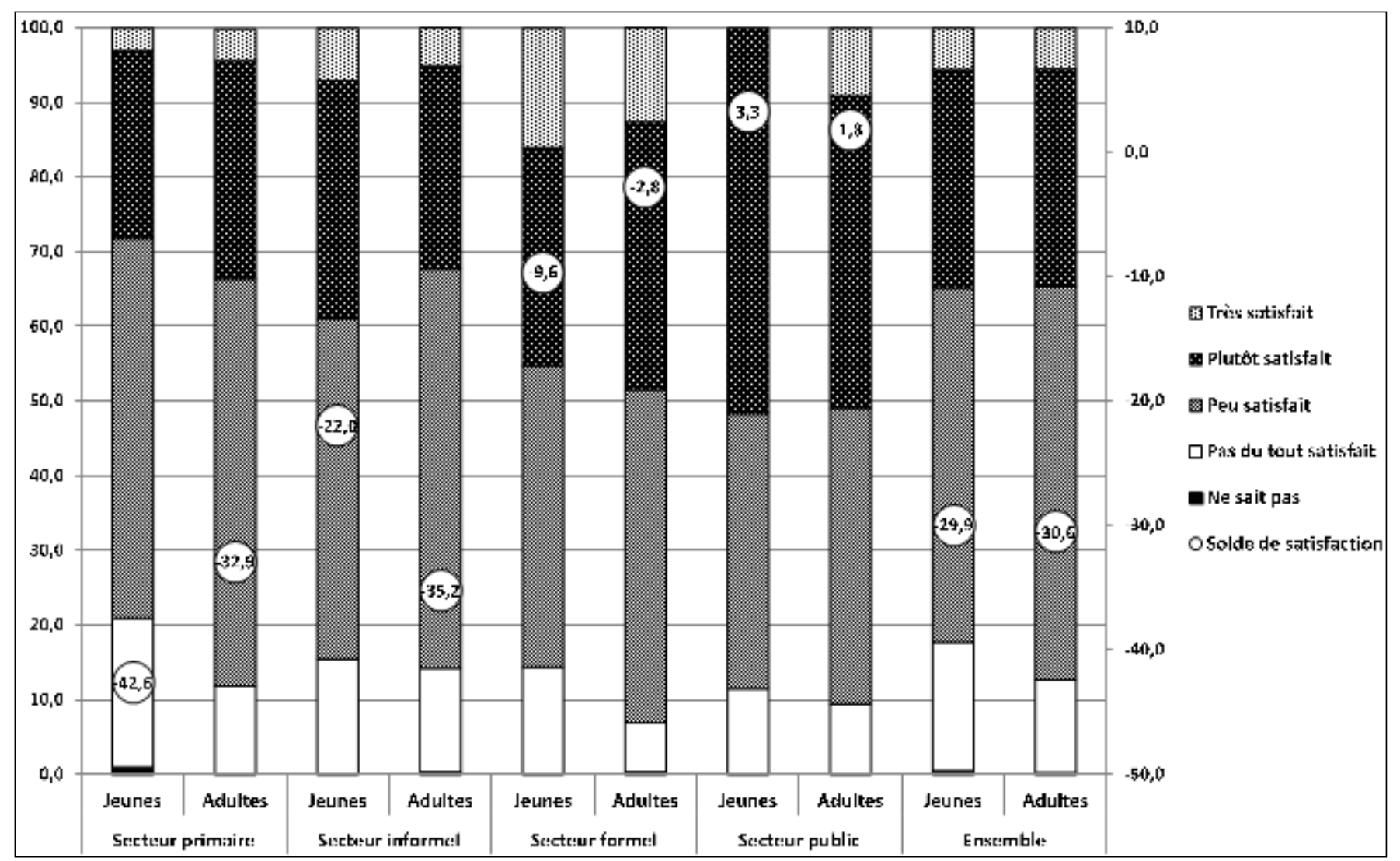

Sources : ECVMAS, 2012, IHSI / DIAL / Banque mondiale ; calculs des auteurs.

\section{IV.- La jeunesse : une et indivisible ? Selon que vous serez puissant ou misérable...}

Jusqu'ici, nous avons considéré les jeunes comme un ensemble homogène et mené nos analyses en comparant les générations. Cette approche, qui a le mérite de donner une vue d'ensemble, peut aussi être trompeuse, pour peu que le destin de la jeunesse ne soit pas le même suivant le type de population considérée. De ce point de vue, deux modèles radicalement différents peuvent être opposés. Dans les pays développés, en France en particulier, la crise a eu pour effet d'accroître les avantages acquis en faveur des jeunes issus des milieux aisés [Baudelot et Establet, 2000]. La dernière enquête PISA sur les acquis scolaires montre que les contreperformances scolaires sont encore plus aigues aujourd'hui [OCDE, 2013]. En revanche, dans les pays d'Afrique sub-saharienne dont se rapproche Haiti en termes de développement, ce sont les plus diplômés qui ont subi le plus fort déclassement, d'une génération à l'autre [Antoine et al., 2001]. Ce résultat paradoxal s'explique par le fait que la dégradation du marché du travail s'est surtout traduit par le gel des embauches dans le secteur formel (principalement public) dont les postes étaient massivement occupés par les enfants originaires des classes moyenne et supérieure. La crise s'est traduite par un ajustement par le bas, qui par ailleurs a eu comme effet secondaire de réduire les inégalités entre classes sociales. A quel modèle se rattache Haïti ? Pour répondre à cette question, trois entrées sont privilégiées : le genre, le lieu de résidence (urbain vs rural) et enfin l'origine sociale des 
individus, cette dernière étant appréhendée principalement par le niveau d'étude du chef de ménage dans lequel vivent les jeunes ${ }^{1}$.

L'analyse en termes de classes sociales ne souffre aucune ambiguïté : ce sont les jeunes originaires des milieux défavorisés qui ont payé le plus lourd tribu (suite) à la catastrophe. Dans un contexte de forte poussée du taux d'emploi des jeunes, celle-ci est d'autant plus forte qu'ils proviennent d'un milieu modeste. A titre emblématique, en 2007 le taux d'emploi des enfants était insignifiant, quel que soit leur origine sociale. En 2012, alors qu'il reste négligeable dans les familles où le chef a été à l'université $(1,8 \%)$, il ne cesse de croître en raison inverse du niveau d'étude du chef, jusqu'à atteindre près d'un enfant sur quatre $(23 \%)$ chez ceux où le chef n'a pas fréquenté l'école. Seul l'effort consacré à la scolarisation des jeunes ne varie pas suivant l'origine sociale. Mais, si les inégalités ne s'accroissent pas, elles se maintiennent à un niveau élevé. Par exemple, alors qu'aux deux périodes à peine $2 \%$ des enfants des classes supérieures ne vont pas à l'école, ils sont $12 \%$ lorsque le chef de ménage n'y est pas allé lui même. Si le différentiel de taux de scolarisation s'est maintenu, en revanche, l'écart en termes de temps consacré à l'éducation formelle s'est creusé. Par exemple chez les enfants, partant d'un niveau équivalent en début de période (environ 12 heures par semaine), il se maintient voire augmente légèrement ( $+1,5$ heures) chez les plus aisés, tandis qu'il se contracte de 4 heures à l'autre extrémité de la hiérarchie sociale.

Plus généralement, tous les indicateurs analysés dans les deux parties précédentes et montrant la dégradation du sort des jeunes (d'une période sur l'autre et par rapport à leurs ainés), sont d'autant plus en recul que l'on descend dans l'échelle sociale. Par exemple, même si les emplois dans le secteur formel sont globalement inaccessibles pour l'ensemble des jeunes, la part du secteur primaire décroît très sensiblement lorsqu'on monte dans la hiérarchie sociale : de $76 \%$ chez les enfants d'un chef jamais scolarisé à seulement $21 \%$ lorsque le chef de ménage a fréquenté l'université. Ce recul des emplois agricoles est compensé à due proportion par la forte croissance des emplois du secteur informel (18\% et $66 \%$ respectivement).

Il n'est pas question de détailler ici tous les indicateurs disponibles illustrant l'accroissement des inégalités suivant l'origine sociale des jeunes si ce n'est pour indiquer qu'il n'en est pas un allant dans le sens d'une amélioration de la position relative des jeunes issus des milieux défavorisés. On se contentera et à titre illustratif de présenter quelques exemples en commençant par l'évolution des rémunérations. Dans l'ensemble la perte de pouvoir d'achat tiré de l'emploi est d'autant plus élevée que l'on se déplace vers le bas de la hiérarchie sociale. Ainsi, chez les jeunes qui bénéficient d'un emploi rémunéré, la baisse moyenne est de $26 \%$ (hors revenus nuls) lorsque le chef de ménage est analphabète. A contrario ils enregistrent un gain de $91 \%$ (hors revenus nuls) lorsque ce dernier a fréquenté l'université (Tableau 5).

Les résultats sont à l'avenant, que l'on considère la rémunération médiane ou que nos comparaisons portent sur l'ensemble de ceux qui ont un emploi rétribué ou non. Cette dynamique différenciée débouche mécaniquement sur une explosion des inégalités de

\footnotetext{
${ }^{1}$ Dans $60 \%$ des cas, les jeunes sont les enfants biologiques du chef de ménage ou de son conjoint. La part des jeunes qui ne sont pas apparentés à ces derniers est faible (\%). Enfin 5\% des jeunes sont eux-mêmes chefs de ménage ou leur conjoint. Dans ce cas nous avons pris le niveau d'étude du jeune lui-même. Par ailleurs niveau de revenu par tête (robustesse consommation par tête pour 2012).
} 
traitement. Alors qu'en 2007 les jeunes d'origine modestes percevaient 33\% de moins que leurs homologues les plus aisés, l'écart est passé à $-85 \%$ en 2012. On notera par ailleurs que le différentiel de rémunération suivant le statut social du chef de ménage est beaucoup plus marqué pour les jeunes que pour leurs ainés. Le taux de sous-emploi global est un bon indicateur synthétique des tensions sur le marché du travail. Ce dernier a augmenté de +27 points chez les jeunes d'origine modeste contre +9 points pour ceux issus de milieux plus aisés, alors même qu'il partait d'un niveau équivalent en 2007.

\section{Tableau 5}

Evolution de l'insertion des jeunes suivant l'origine sociale 2007, 2012

\begin{tabular}{|c|c|c|c|c|c|c|}
\hline \multirow[b]{2}{*}{$\begin{array}{c}\text { Seulement à l'école (10-14 } \\
\text { ans) }\end{array}$} & \multicolumn{6}{|c|}{ Niveau de scolarité du chef de ménage } \\
\hline & Aucun & $\begin{array}{l}\text { Primaire } \\
\text { incomplet }\end{array}$ & $\begin{array}{c}\text { Collège } \\
\text { incomplet }\end{array}$ & $\begin{array}{c}\text { Lycée } \\
\text { incomplet }\end{array}$ & Supérieur & Ensemble \\
\hline En $2012(\%)$ & 69,4 & 73,7 & 80,0 & 81,3 & 95,6 & 75,1 \\
\hline Evolution 2007-2012 (\%) & $-20,1$ & $-18,7$ & $-14,1$ & $-17,1$ & $-2,5$ & $-16,8$ \\
\hline \multicolumn{7}{|l|}{ Taux d'emploi } \\
\hline En $2012(\%)$ & 33,7 & 27,5 & 25,3 & 23,9 & 16,8 & 28,4 \\
\hline Evolution 2007-2012 (\%) & $+157,3$ & $+174,5$ & $+104,1$ & $+119,7$ & $+88,9$ & $+142,8$ \\
\hline \multicolumn{7}{|c|}{ Rémunération mensuelle (y compris les revenus nuls) } \\
\hline En $2012(\%)$ & 706 & 809 & 2161 & 2669 & 4648 & 1296 \\
\hline Evolution 2007-2012 (\%) & $-73,1$ & $-75,8$ & $-37,3$ & $-37,7$ & $+18,0$ & $-58,6$ \\
\hline \multicolumn{7}{|l|}{ Taux de sous-emploi global } \\
\hline En $2012(\%)$ & 92,7 & 93,2 & 84,3 & 74,2 & 78,8 & 88,8 \\
\hline Evolution 2007-2012 (\%) & $+26,7$ & $+28,1$ & $+17,7$ & $+8,5$ & $+9,4$ & $+22,8$ \\
\hline
\end{tabular}

Sources : EEEI, 2007, IHSI / DIAL ; ECVMAS, 2012, IHSI / DIAL / Banque mondiale ; calculs des auteurs.

L'évolution des conditions de travail conforte et aggrave le diagnostic. Alors que le travail irrégulier représente environ un quart des emplois chez les jeunes (un peu moins en 2007), cette forme d'emploi précaire se maintient à un niveau faible chez les enfants des familles les plus éduquées (13\%), alors qu'elle augmente brutalement de $22 \%$ à $33 \%$ en 5 ans chez ceux qui sont issus de familles modestes. Même constat pour le lieu d'exercice de l'emploi. En 2012, 6\% des jeunes travailleurs dont le chef n'a aucun diplôme ont la chance de jouir d'un local professionnel. Cette proportion monte à $45 \%$ pour ceux dont le père a fréquenté le supérieur. Encore une fois, la précarisation pèse avant tout sur les plus modestes avec une baisse de 10 points vs 4 points, qui exprimée en pourcentage est encore plus spectaculaire ($62 \%$ vs $-8 \%)$.

Le niveau d'éducation du chef de ménage est un bon indicateur de stratification sociale comme en attestent les résultats présentés ci-dessus. Cependant, pour tester la robustesse de nos conclusions, nous avons élaboré un certain nombre de critères alternatifs pour identifier le milieu d'origine. De façon tout à fait classique, nous avons subdivisé les ménages en quintiles de revenus par tête, les revenus incluant toutes les rémunérations tirées des emplois (aussi bien principaux que secondaires) de tous les membres du ménage, ainsi que des transferts reçus. Cette mesure est nécessairement imparfaite. D'abord, parce que le montant exact des revenus est toujours difficile à saisir ; ensuite, parce que ces derniers évoluent dans le temps 
et nous ne pouvons garantir que les ménages observés en 2012 se situaient dans le même quintile en 2007. Tous les indicateurs liés à la scolarisation et au marché du travail ont été calculés suivant cette nouvelle typologie. Nous ne pouvons pas reproduire l'ensemble des résultats ici faute de place $^{2}$, mais la principale conclusion est que le constat obtenu avec le niveau d'éducation du chef de ménage reste valide. Afin de lever l'hypothèque concernant les problèmes potentiels de mesure des revenus, nous avons reproduit les analyses par quintile de consommation par tête (disponible seulement en 2012). Les résultats précédents en sortent confortés.

Pour finir cette partie, nous avons cherché à savoir si le poids disproportionné porté par les jeunes des milieux défavorisés face aux chocs négatifs avait une portée plus générale. Plus précisément, reflète-t-il un mode de régulation de la société haïtienne, où les groupes socialement les plus fragiles, parce qu'ils possèdent moins de capitaux (économique, social et culturel), ou parce qu'ils sont discriminés, ou les deux, sont toujours les principales victimes lorsque l'environnement se dégrade. Parmi les groupes de population soumises au risque de déclassement, nous en avons déjà repérés deux, à savoir les femmes et les ruraux [Justesen et Verner, 2007 ; Roubaud et al., 2013]. Ce résultat obtenu en population générale s'applique-t-il aussi parmi les jeunes ? La réponse ne souffre aucune discussion : lorsqu'on est jeune, il vaut mieux être un homme résidant en ville qu'une femme à la campagne (résultats non reportés mais disponibles sur demande auprès des auteurs). Chacune de ces trois dimensions (groupes sociaux, genre, milieu de résidence) sont associés à un effet propre, que le passage de la statistique descriptive aux modèles de régressions, toute choses égales d'ailleurs, permet d'isoler.

\section{Conclusion}

Grâce à un jeu de deux enquêtes de grande envergure réalisées à cinq ans d'intervalle avec l'appui scientifique des auteurs, nous avons pu établir un diagnostic de la situation de la jeunesse avant et après le séisme. En nous centrant sur le marché du travail (et plus accessoirement sur la scolarisation), nous traitons d'une dimension essentielle du passage à l'âge adulte, à côté des processus d'autonomisation matrimoniale et résidentielle. De cette étude basée sur des données à ce jour encore inédites, nous retiendrons trois résultats principaux.

En premier lieu, les chocs négatifs subi par les Haïtiens entre 2007 et 2012, se sont traduits par un mécanisme d'ajustement à la baisse extrêmement brutal dont la charge porte de façon disproportionnée sur les plus faibles : les jeunes plus que les adultes (et parmi les jeunes, les moins âgés d'entre eux), les femmes plus que les hommes, les ruraux plus que les urbains, les pauvres plus que les riches, les stigmates sociaux se cumulant les uns aux autres. Dans ce contexte, si les politiques et l'aide internationale ont probablement contribué à atténuer le choc pour tous, elles n'ont pas réussi à enrayer une dynamique structurellement inégalitaire, un révélateur éclatant et cruel des fondements de la société haïtienne, pour le pire.

En deuxième lieu, les mécanismes d'ajustement ont pris des formes pas nécessairement attendus. Alors qu'on aurait pu augurer d'une baisse de la scolarisation et une explosion du

\footnotetext{
${ }^{2}$ Les résultats sont disponibles auprès des auteurs.
} 
chômage, on observe au contraire une mise au travail généralisée des jeunes, concomitante d'une légère amélioration de l'accès à l'école. Cependant, la qualité des emplois occupés s'est profondément dégradée et ce dans toutes ses dimensions (rémunération, conditions de travail, protection, etc.). De plus, les inégalités ont bondi alors qu'elles étaient déjà parmi les plus élevées au monde. S'il n'est pas possible d'attribuer cette dynamique régressive au seul séisme de 2010, les changements observés sont d'une telle ampleur qu'ils ne peuvent être le résultat que d'un choc sans précédent. Le séisme apparait donc comme le candidat le plus probable, même s'il n'est pas le seul. On peut même penser que le séisme a constitué un facteur de déstabilisation majeur contribuant à bouleverser un équilibre déjà fragile, et de ce fait a accru l'impact négatif des chocs subséquents.

En troisième lieu, les jeunes ne semblent pas plus désespérés que leurs ainés, les deux groupes se montrant aussi insatisfaits de leur emploi l'un que l'autre. Ce résultat déjoue les attentes. Il est d'autant plus paradoxal, que l'emploi des jeunes s'est le plus détérioré et qu'en général leurs aspirations plus exigeantes et leurs ambitions plus élevées. Ce phénomène demande des recherches spécifiques pour en comprendre les ressorts. Mais plus concrètement, il suggère que les jeunes haïtiens ne sont pas porteurs d'un mécontentement social spécifique, et qui serait susceptible de déboucher sur des mouvements de contestation politique ou de violence généralisée. Il convient cependant de rester prudent sur ce point, l'histoire ayant montré que les réactions de la jeunesse à leur environnement étaient rarement prévisibles.

Si les risques d'explosion sociale entrainée par la jeunesse haïtienne semblent a priori écartés, le sort inique qui lui est fait doit être corrigé de manière urgente. Il s'agit bien sûr d'une question de justice sociale, mais au-delà c'est l'avenir du pays qui est en jeu. En effet, le sacrifice de la génération montante risque d'entretenir un cercle vicieux intergénérationnel gageant la croissance économique de long terme. Un tel engrenage installerait durablement Haïti dans une trappe à pauvreté à laquelle il deviendrait difficile d'échapper.

Cet article n'est pas le lieu pour tenter de proposer une liste de mesures à prendre. Deux points nous semblent néanmoins pouvoir être très directement tirés de nos analyses. D'abord, il est urgent de mettre en place des politiques spécifiques et à grande échelle ciblant les jeunes, ce qui n'est pas le cas actuellement. Ensuite, des enseignements peuvent être tirés du hiatus observé entre la stratégie éducative des familles qui acceptent des sacrifices pour maintenir à tout prix leurs enfants à l'école, et la décision de leur imposer une mise au travail précoce. La première différence entre ces deux types de comportement est que l'un est choisi alors que l'autre est imposé. La seconde différence provient du fait que la demande des ménages en matière d'éducation rencontre une offre (un système scolaire plus ou moins fonctionnel), ce qui n'est pas le cas pour le marché du travail. Il existe donc une marge de manœuvre pour des politiques publiques visant à stimuler la création d'emplois de qualité. Cette marge est étroite, mais tout effort dans ce sens ne pourrait être qu'un pas dans la bonne direction.

\section{Bibliographie}

Antoine P., Razafindrakoto M., Roubaud F. [2001], "Contraints de rester jeune ? Evolution de l'insertion dans trois capitales africaines : Dakar, Yaoundé, Antananarivo ", 
in Collignon R., Diouf M. (dir.), «Les jeunes: hantise de l'espace public dans les sociétés du Sud », Autrepart No. 18, p.17-36.

BAD, CEA, OCDE, PNUD [2012], «Promouvoir l'emploi des jeunes », in Perspectives économiques de l'Afrique 2012, Chapitre 6, Editions de l'OCDE, Paris, pp..

Baudelot Ch., Establet R.[2000], Avoir 30 ans en 1968 et en 1998, Le Seuil, Paris.

BiLHAM R. [2010], « Lessons from the Haiti earthquake», Nature Vol. 463(18) 878-879.

Camp Coordination And CAmp Management (CCCM) [2013], Cluster Haiti: Updated list of IDP sites of the DTM, October 2013

[Datafile] http://groups.google.com/group/cccmhaiti?pli=1, consulté le 14 novembre 2013.

CAVAllo E., NoY I. [2009], « The economics of natural disasters-A survey », IADB Working Paper $n^{\circ} 09-19$.

Cayemittes M., Fatuma Busangu M., Bizimana J., Barrere B., Severe B., Cayemittes

V., Charles E. [2013], Enquête Mortalité, Morbidité et Utilisation des Services, Haïti, 2012.

Calverton, Maryland, USA : MSPP, IHE et ICF International.

Chauvel L. [1998], Le destin des générations. Structure sociale et cohortes en France au XXème siècle, PUF, Paris.

DIAL [2007], « Youth and Labour Markets in Africa: A Critical Review of Literature », $\begin{array}{lllll}\text { Document de } & \text { Travail } & \text { DIAL, } & \text { No } & \end{array}$ (www.dial.prd.fr/dial_publications/PDF/Doc_travail/2007-02.pdf) et Working Paper No 49, Département de la Recherche, Agence Française de Développement (AFD), 67 p., octobre (www.afd.fr/jahia/Jahia/home/publications/documentsdetravail/pid/3832)

Doocy S., CHEREwICK M., KIRSCH T. [2009], « Mortality following the Haitian earthquake of 2010: a stratified cluster survey ». Population Health Metrics 2013, 11:5/1478-7954$11-5$

EM-DAT. The OFDA/CRED International Disaster Database, 2011. Hosted by Université Catholique de Louvain, Brussels, Belgium. Haiti Country Profile. http://www.emdat.be. Consulté le 23 janvier 2011.

Galland O. [1997], Sociologie de la jeunesse, Paris, A. Colin, 248 p.

Galland O. [1999], Les jeunes, Paris, Repères, La Découverte, 124 p.

Gauthier M., Guillaume J.F. (s.d.], Définir la jeunesse? D'un bout à l'autre du monde. Collection culture et société. Les éditions de l'IQRC, Québec, p. 95-106.

Heger M., Julca A., PAdDison O. [2008], Analyzing the Impact of Natural Hazards in Small Economies: The Caribbean Case. UNU/WIDER Research paper 2008/25.

Justesen M., Verner D. [2007], "Factor Impacting Youth Development in Haiti", World Bank Policy Research Working Paper $n^{\circ} 4110$, Washington D.C., Janvier. 
Kolbe A., Hutson R., Shannon H., Trzcinski E., Miles B., Levitz N. [2010], Mortality, crime and access to basic needs before and after the Haiti earthquake: a random survey of Port-au-Prince households. Medicine, Conflict and Survival 2010, 26(4):281-297.

Lunde H. [2012], The violent lifeworlds of young Haitians. Gangs as livelihood in Port-auPrince ghetto, The Haiti Youth Project, Fafo, Oslo.

Lunde H. (DIR.) [2010], Haiti Youth Survey 2009. Volume II: Analytical Report, The Haiti Youth Project, Fafo, Oslo.

Mueller V., Quisumbing A. [2009a], Natural Disasters and their Labor Market Consequences: Evidence from the 1998 flood in Bangladesh, IFPRI, mimeo.

MUELLER V., OSGOOD D. [2009b], Long-term impacts of droughts on labor markets in developing countries: Evidence from Brazil, Journal of Development Studies 45(10): $1-13$.

OCDE [2013], Principaux de l'enquête PISA 2012 : ce que les élèves de 15 ans savent et ce qu'ils peuvent faire avec ce qu'ils savent, Editions de l'OCDE, Paris.

RAZAFINDRAKOTO M., RoubaUd F. [2013], « La satisfaction dans l'emploi. Une mesure de la qualité de l'insertion professionnelle en regard des aspirations », in De Vreyer P., Roubaud F. (dir.), Les marchés urbains du travail en Afrique sub-saharienne, chapitre 3, Editions de l'IRD, Marseille, p.125-150).

RAZAFINDRAKOTO M., RoubAud F., WACHSBERGER J.-M. [2012], « Travailler dans le secteur informel : choix ou contrainte ? Une analyse de la satisfaction dans l'emploi au Vietnam », in Cling J.-P., Lagree S., Razafindrakoto M. et Roubaud F. (dir.), L'économie informelle dans les pays en développement, chapitre 1.2, Editions de l'AFD, Paris, p.47-66.

Roubaud F., Torelli C., Zanuso C. [2013], Enquête sur les Conditions de Vie des Ménages Après Séisme ECVMAS-Haïti 2012 : le marché du travail : situation en 2012 et évolution depuis 2007, IHSI / DIAL, Paris - Port-au-Prince.

Schwartz T., PIERRE Y.F., CALPAS E. [2011], Building Assessments and Rubble Removal in Quake-Affected Neighborhoods in Haiti. BARR Final Survey Report. Released May 13, 2011.

Skoufias E. [2003], Economic Crises and Natural Disasters: Coping Strategies and Policy Implications. World Development 31(7), 1087-1102.

YAMAUCHI F., YOHANNES Y., QUISUMBING A. [2009], Natural disasters, self-Insurance, and human capital investment: Evidence from Bangladesh, Ethiopia, and Malawi, IFPRI Discussion Paper 881. Washington, D.C. 